\title{
Statistical model of metal removal removed from product surface under the influence of abrasive particle flow
}

\author{
Georgi Tzordanidi ${ }^{1}$, Oksana Pyatnitzkaya ${ }^{1}$, Elena Fisunova $^{1}$, Tatyana Lavrenova ${ }^{1,{ }^{*}}$, and \\ Olga Baryshnikova ${ }^{1}$ \\ ${ }^{1}$ Don State Technical University, Gagarin sq., 1, Rostov on Don, 344003, Russia
}

\begin{abstract}
A model is proposed that describes the forming processes of metal volume removed from the product surface during processing with a free abrasive flow, taking into account the random nature of abrasive particle impact on the surface and the possibility of implementing acts of elastic and plastic displacement of material or micro-cutting.
\end{abstract}

\section{Introduction}

Numerous studies of the processes of abrasive wear and finishing processing with a fine abrasive have established that the amount of stress that occurs in the contact zone of a single particle with flow of the product depends on the energy of the particle and its shape. The latter are random in nature.

The penetration depth of the particle into the surface-h is determined by its energy, and the radius of rounding of the vertex $\varrho$ determines the value of the contact spot, so $h / \varrho$ is a dimensionless criterion for similarity of stresses occurring in the contact zone.

According to the classification proposed by I. V. Kragelsky [1], the following processes can occur in the contact zone:

- Elastic repulsion of metal when the stress does not exceed the yield point $\sigma_{\mathrm{T}}$ and there is a destruction of the surface as a result of multi-cycle friction fatigue.

- Plastic displacement, in which contact stresses reach $\sigma_{\mathrm{T}}$, but the metal is not destroyed by a single impact, and its removal is the result of low-cycle friction fatigue.

- Micro-cutting - at stresses exceeding $\sigma_{\mathrm{T}}$, destruction occurs with a single impact [2].

Each of the considered types of destruction can be matched with the number of contacts $\mathrm{N}$ : for elastic displacement, $\mathrm{N}=106-1010$, for plastic $\mathrm{N}=2-106$, and $\mathrm{N}=1$ for microcutting.

When talking about the number of cycles to failure in multi-cycle and low-cycle fatigue, it should be remembered that in the processes of abrasive wear or processing, it is equal to the number of consecutive loads of the same micro-volume of metal. Therefore, the possibility of implementing these destruction processes depends on the geometric probability of repeated acts of impact of abrasive particles on the same area of the product surface, equal to the area of the contact spot [3].

*Corresponding author: bys_ka87@mail.ru 


\section{Goal and objectives}

This article attempts to describe random processes occurring in the contact zone of free abrasive particles with the surface of the product, leading to the separation of metal micro volumes, from the position of the probability torus. At the same time, it was assumed that such characteristics of the processing process as the degree of fixation of abrasive particles in the flow, the density of contact of particles, measured by the act number of their interaction with the surface, the size of the particles and the radius of rounding of the vertices, the strength indicators of the processed material [4].

\section{Research methods and results}

The analysis of impact traces of abrasive grains left by them on the polished samples revealed two conjugate sections - active $\mathrm{L}_{\mathrm{l}}$, corresponding to the introduction of the grain into the surface and passive $\mathrm{L}_{\mathrm{II}}$, the length of which depends on the degree of fixation of the particles in the flow Fig. 1.

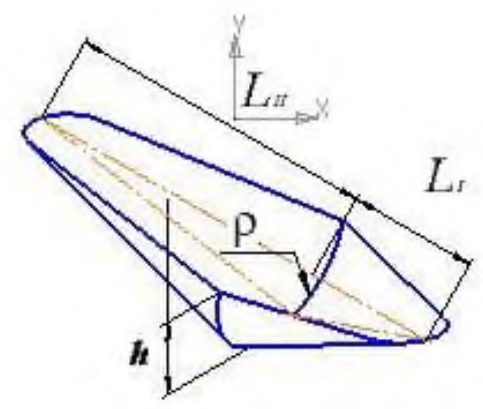

Fig. 1. Parameters of a single trace

In all cases, the process of the hole formation was accompanied by plastic repulsion of metal along its entire perimeter in the form of a roller heigh $h$ ' Fig.2.

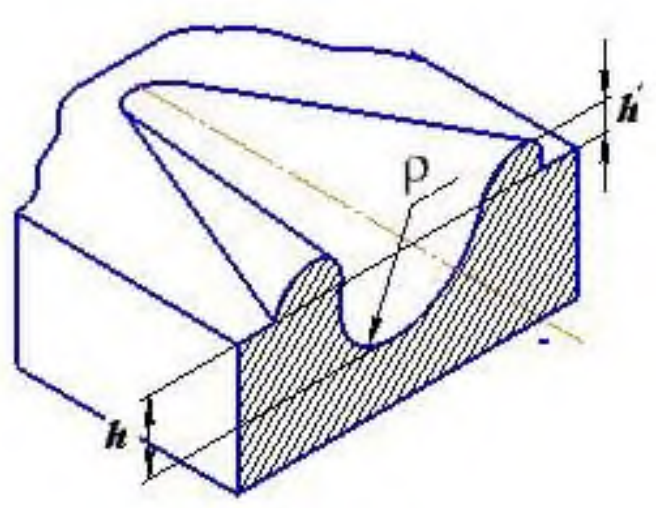

Fig.2. Plastic repelling of metal 
$W=W_{I}+W_{I I}=\pi h^{2}\left(\rho-\frac{h}{3}\right)+\frac{f_{C}}{3} \cdot L a, m^{3}$ According to [5], the volume of the hole $\mathrm{W}$ can be defined as the sum of the volumes of the active and passive sections,

$$
W=W_{I}+W_{I I}=\pi h^{2}\left(\rho-\frac{h}{3}\right)+\frac{f_{C}}{3} \cdot L a, m^{3}
$$

where $\mathrm{fc}$ is the area of the circular segment that separates the active and passive sections.

$$
f_{c}=\rho^{2}\left[\arcsin \sqrt{1-\left(1-\frac{h}{\rho}\right)^{2}}-\left(1-\frac{h}{\rho}\right) \sqrt{1-\left(1-\frac{h}{\rho}\right)^{2}}\right]
$$

The volume of metal removed by micro-cutting can be calculated according to the following formula:

$$
\mathrm{W}_{\mathrm{p}}=\mathrm{W}^{*} \mathrm{~K}_{\mathrm{c},} \mathrm{m}^{3}
$$

Where $K_{c}$ is the chip formation coefficient [6].

The volume of metal removed as a result of plastic displacement in the form of a roller surrounding the hole can be calculated according to the following formula:

$$
W_{g}^{\prime}=\pi \rho^{3}\left(\frac{h}{\rho}\right)^{2}, m^{3}
$$

From the point of view of reliability theory, the process of abrasive destruction of the processed product surface is similar to the scheme of accumulated damages, by which we understand the acts of interaction that lead to metal removal, referred to in this context as failure [7]. This scheme describes the Gamma distribution of uptime T. The density function of this distribution has the form:

$$
f(T)=\frac{1}{\Gamma_{(2)}} \cdot \lambda^{2} T^{2-1} \cdot e^{-\lambda T}
$$

where 2 is the number of damages that cause failure.

The parameter 2, taking integer values, describes both acts of micro-cutting and separation of micro-volumes due to low-and multi-cycle fatigue, characterized by the presence of a latent period [8-10]. In the case where $2=1$, the value of the density function corresponds to the time interval between two failures at a single impact, or, what is the same, between two acts of micro-cutting

$$
f(T)=\lambda e^{-\lambda T}
$$

Considering the flow of random events consisting of implementations of micro-cutting acts, and assuming that the Commission of these acts is independent of the time interval between them, from the beginning of the countdown, from the possibility of implementing these acts from subsequent or previous ones, we came to the conclusion that such a flow of events is Poisson, and the time interval between two consecutive events obeys the law described by equality (5). Parameter

$$
\lambda=\frac{n_{p}}{n_{g}}=\frac{P(0,04<h / \rho<1)}{P(0<h / \rho<0,04)}
$$

describes the relationship between the probabilities of micro-cutting and deforming processes calculated for values that determine the transition from crumpling to cutting [1113]. Conditionally dividing the acts of plastic deformation into passive ones, which were 
not implemented due to the implementation of the act of micro-cutting, and active ones, which led to destruction after reaching a certain number of cycles $\mathrm{N}$, metal removal-W is the sum of volumes removed as a result of fatigue processes taking into account the imposition of micro-cutting acts- $W_{g}$, and destruction only from micro-cutting- $W$

$$
W=W_{p}+W_{g}=\left(W_{g}^{\prime} \frac{n_{p}}{e^{\lambda N}-1}+W_{p}^{\prime} \cdot n_{p}\right) \cdot \gamma
$$

or taking into account (2.3)

$$
W=P\left(0,004<\frac{h}{\rho}<1\right) \cdot z_{0} \cdot \gamma \cdot\left(\frac{W_{g}^{\prime}}{e^{\lambda N}-1}+W_{p}^{\prime}\right),
$$

where $Z_{0}$ is the total number of contacts of abrasive particles during the considered processing time;

$\mathrm{Wg}^{\prime}, \mathrm{Wp}^{\prime}$ - individual volumes removed as a result of fatigue failure and micro-cutting; $\gamma$ is the density of the processed material.

The term $\frac{W_{g}^{\prime}}{e^{\lambda N}-1}$ in the expression (9) describes the polydeformational component of metal removal only if the number of abrasive particle contacts with a surface area that restricts the unit volume of $\mathrm{Wg}^{\prime}$ is equal to the number of cycles to destruction at the appropriate level of contact stresses, depending on the ratio $\frac{h}{\rho}$.

Having the value $\mathrm{N}$ for cyclic deformation in the plastic region, it is possible to evaluate the possibility of polydeformation destruction processes by solving the following problem:

considering the contact spot area and the projection area of the particle on the treated surface, calculate the probability that when $Z$ hits the abrasive particles in the square of the package, any point of it will be covered by the contact spot exactly $\mathrm{N}$ times [14].

Make the following assumptions:

- projections of particles on the surface and contact spots are flat circles with diameters $D$ and $d_{n}$ respectively;

- the particles in the stream are Packed in a square with side $D=L_{3}$;

- contact spots are the same for all particles.

The task is divided into two parts. The first of them is the determination of the covering geometric probability of any point of the package square with a contact spot solved by Garwood

$$
\rho=\frac{\pi d_{n}^{2}}{4 D^{2}+8 d_{n} D+\pi d_{n}^{2}}
$$

The second consists in finding the probability of repeating the event exactly $\mathrm{N}$ times in $\mathrm{Z}$ independent experiments, that is, it reduces to the Bernoulli scheme

$$
\begin{aligned}
P= & N \\
& C \\
Z & \rho^{N} \cdot(1-\rho)^{Z-N}
\end{aligned}
$$

For sufficiently large $\mathrm{Z}$ values, calculating $\mathrm{P}$ using the formula (11) becomes inconvenient. Therefore, we will use the dependency to calculate the probability of covering each point of the surface of the part with contact spots at least $K_{l}$ and no more than $K_{2}$ times

$$
P\left(K_{1} \leq K \leq K_{2}\right) \approx \Phi\left(\frac{K_{2}-n \rho}{\sqrt{n \rho q}}\right)-\Phi\left(\frac{K_{1}-n \rho}{\sqrt{n \rho q}}\right)
$$


where $\Phi(x)=\frac{1}{\sqrt{2 \pi}} \int_{0}^{x} e-\frac{t^{2}}{2} d t$ - Laplace function, a $q=1-\rho$ there is a probability of the opposite event $\rho$.

Since the destruction of a unit volume occurs if the number of impacts is at least N, let's put $K_{2}=\mathrm{Z}=\mathrm{n}$ and $K_{1}=\mathrm{N}$ in the expression (12), then

$$
P(Z \geq N)=\Phi\left(\frac{Z-Z \rho}{\sqrt{Z \rho q}}\right)-\left(\frac{N-Z \rho}{\sqrt{Z \rho q}}\right)
$$

Given that $1-\rho=q$, after the transformations, we finally get

$$
P(Z \geq N)=\Phi\left(\sqrt{\frac{Z q}{\rho}}\right)-\Phi\left(\frac{N-Z \rho}{\sqrt{Z \rho q}}\right)
$$

Believing in (13) based on the principle of practical certainty $P(Z \geq N)=0,95$, it is easy to determine $Z$ that corresponds to the conditions of a specific task. In the case $Z>$ 1 , and since $\sqrt{\frac{q}{\rho}}>5$ (according to the results of our experiments), the first term in (13) is always 0,5 .

Therefore, for $P=0,95 \Phi\left(\frac{N-Z \rho}{\sqrt{Z \rho q}}\right)=-0,45$. Hence $\mathrm{Z}$ is a real positive root that converts the equation into an identity

Returning to the relationship (9), we note that the floor and the deformation component in the removal of metal should be taken into account only if

$$
N<Z \leq Z_{0} \text {, and therefore } \frac{W_{g}}{e^{\lambda N}-1} \neq 0
$$

It is obvious that when $Z_{0}<Z$ the completion of the floor and deformation processes by destruction becomes impossible and the expression (9) takes the form

$$
W=P\left(0,04<\frac{h}{\rho}<1\right) \cdot \gamma \cdot Z_{0} \cdot W_{\rho}^{\prime}, \mathrm{kg} .
$$

Let's express the total number of contacts for the considered period of time $-Z_{0}$ in terms of the contact density- $\widehat{m}$, equal to the number of abrasive particles affecting the selected elementary surface area per unit of time.

$$
Z_{0}=\widehat{m} \cdot T \cdot S, c^{-1} \cdot m m^{-2},
$$

where $\mathrm{T}$ is the duration of processing;

$\mathrm{S}$-is the area of the processed surface.

Substituting (16) in (9) and dividing both parts by $\mathrm{T}$, we get an expression for the processing performance $\mathrm{Q}$, which can serve as a statistical model of the metal removal process when processing in a free abrasive environment $[15,16]$

$$
Q=P\left(0,04<\frac{h}{\rho}<1\right) \cdot \gamma \cdot \widehat{m} \cdot S \cdot\left(\frac{W_{g}^{\prime}}{e^{\lambda N}-1}+W_{\rho}^{\prime}\right), k g / s
$$

\section{Conclusions}


1. The expression for the processing process productivity is universal, due to the similarity of the formation processes of single traces left on the product surface by a single abrasive grain in various technological processes.

2. The Volumes of metal in individual acts of micro-cutting and post-formation destruction $\mathrm{W}_{\mathrm{p}}$, and $-\mathrm{W}_{\text {д }}$ can be calculated using the proposed dependencies.

3 . In experiments with a black $54 \mathrm{C}$ silicon carbide grinding mill with a grain size of 125,63 and 40 according to All-Union State Standard 3647-80, the error in estimating metal removal for this model does not exceed $20 \%$ and the less, the higher the grain size of the abrasive material.

\section{References}

1. I. V. Kragelsky, Friction and wear. - 2nd ed., republished. Moscow: Manufacturing, 480 (2011)

2. M. A. Tamarkin, E. E. Tishchenko, A. S. Melnikov, Technological bases of optimization of processes of detail finishing and strengthening processing in granulated working environments: Proceedings of the X International scientific and practical conference, ed. Kemerovo: KuzSTU, 555-563 (2019)

3. A. N. Unyanin, P. R. Finageev, Statistical analysis of the mechanical processing process with time-varying parameters, High technologies in mechanical engineering: Materials of the XVII All-Russian scientific and technical conference. Samara: Samar, State Technical University, UN-t, 33-35 (2018)

4. E. Yu. Krupenya, A. P. Shishkina, M. A. Boyko, Determination of vibration separation performance of part surface with stone organic media, Proceedings of the International scientific and technical conference dedicated to the 90th anniversary of the Honored worker of science and technology of the Russian Federation, Doctor of Technical Sciences, Honorary Professor of DSTU A. P. Babichev (Rostov-on-don, February 2728, 2018); Don State Technical University. UN-t, Electron. The test is given. Rostovon Don: DSTU 135-138 (2018)

5. A. P. Babichev, Some questions of vibrating treatment theory for maching parts and tools.-in Book: Position and perspectives of productive investigation for treatment by vibration.Rostov n/D, 3-9 (2012)

6. J. J. Gilman, Trans. Amer. Inst. Min. a. Metall. Engrs., 191, 1148 (2014)

7. G. K. Lipsky, B. T. Sheftel, Researches of radial vibration for the bearing in forms in rolling surface by spectral analyses method.-Bearing production, 6, 39 (2016)

8. A. P. Babichev, I. A. Babichev, The basics of vibration technology. Rostov-on-Don: Publishing Center DGTU, 694 (2008)

9. A. P. Babichev, P. D. Motrenko, S. A. Kostenkov and others, Tool support for part processing processes in granular media. - Rostov-on-Don: Ed. DSTU Center, 267 (2011)

10. M. P. Piskus, E. R. Parker. Trans. Amer. Inst. Min. a. Metall. Engrs., 191, 792 (2013)

11. Yu. P. Ankudimov, Yu. G. Chernega, I.V. Sadovaya, The use of integrated energy exposure to modify the surface layer of steel parts by vibration processing. Hardening technologies and coatings, 8, 128 (2015)

12. P. V. Melentjeva, Approximate Calculation. Moscow, Physmathstpubl, 388 (2010)

13. I. A. Babichev, A. P. Babichev, V. V. Ivanov and others, Vibration mechano chemistry in the processes of hardening treatment and coating of machine parts: monograph. Rostov-on-Don (2014) 
14. J. J. Gilman, Amer. Soc. Test. Mater., Spes. Techn., 3, 171 (2010)

15. 1. E. N. da C. Andrade, C. Henderson, Phil. Trans., 244, 177 (2014) 